\title{
Physical treatment of fever
}

\author{
Edward Purssell
}

\begin{abstract}
Fever is a common symptom of childhood illness, and much time and effort is spent in the pursuit of reducing high temperature. Although antipyretic drugs are the main form of treatment, this report considers the part that physical treatments might play in reducing the temperature of febrile children. Such treatments include tepid sponging, removing clothing, and cooling the environment. Of these treatments, tepid sponging has been studied most extensively, as an addition to paracetamol, but seems to offer little advantage over paracetamol alone. It is likely that other methods might be equally ineffective because they all rely on similar methods of heat loss.

(Arch Dis Child 2000;82:238-239)
\end{abstract}

Keywords: fever; physical treatments; tepid sponging; antipyretic drugs

Although the mainstays of antipyretic treatments are drugs such as paracetamol and ibuprofen, physical methods are also used. These include tepid sponging, removing clothing, and cooling the environment with fans and improved ventilation. The most extensively studied of these is tepid sponging, the aim of which is to reduce temperature by conduction of heat from the skin into the cooler water. The sponging action ensures that the water film is constantly moving, replacing the water in contact with the skin with a fresh layer of cooler water, thus maximising heat conduction. Convection currents and evaporation further enhance heat loss. ${ }^{1}$

Florence Nightingale School of Nursing and Midwifery, King's College London, James Clerk Maxwell Building, 57 Waterloo Road, London SE1 8WA, UK

E Purssell

Correspondence to: Mr Purssell

\section{Efficacy}

The effectiveness of tepid sponging as a treatment alongside paracetamol varies between studies (table 1), with some finding that it is of no benefit, ${ }^{23}$ whereas others suggest that it is helpful. ${ }^{45}$ However, even where a positive effect is seen with the addition of sponging to paracetamol, the difference in temperature reduction between those receiving and not Accepted 26 October 1999 receiving the sponging is small; at one hour, the

Table 1 Treatments used in studies comparing paracetamol and sponging with paracetamol alone

\begin{tabular}{llll}
\hline Paracetamol dose & Water temperature & Sponging time & $\begin{array}{l}\text { Temperature } \\
\text { difference } \\
\text { (reference) }\end{array}$ \\
\hline $5-10 \mathrm{mg} / \mathrm{kg}$ & "Neutral" & 20 minutes & $0.2^{\circ} \mathrm{C}^{2}$ \\
$15 \mathrm{mg} / \mathrm{kg}$ & $31.1-33.3^{\circ} \mathrm{C}$ & 15 minutes & $0.8^{\circ} \mathrm{C}^{3}$ \\
$120 \mathrm{mg} \mathrm{(<1} \mathrm{year)}$ & Below body temperature & $10-20$ minutes & $0.1^{\circ} \mathrm{C}^{4}$ \\
$240 \mathrm{mg} \mathrm{(>1} \mathrm{year)}$ & $29-30^{\circ} \mathrm{C}$ & Until $<38^{\circ} \mathrm{C}$ & Not reported \\
$10-15 \mathrm{mg} / \mathrm{kg}$ & &
\end{tabular}

${ }^{\star}$ Difference in temperature between treatments at one hour; temperature fall greater with treatments using sponging and paracetamol in all cases. mean difference in temperature reduction of the three studies reporting this figure was $0.4^{\circ} \mathrm{C} .^{2-4}$ The clinical importance of temperature differences such as these is marginal, particularly when the time involved in sponging is considered.

It has been hypothesised that physical cooling methods might be more effective in tropical climates, and although sponging as a treatment has been demonstrated to be effective, ${ }^{5}$ a study using sponging as a monotherapy has shown that although it initially led to a greater fall in temperature, at one hour paracetamol was a superior treatment. ${ }^{6}$ This suggests that even in hot, humid climates, although radiation and evaporation are important in the immediate loss of heat, this is only a short term effect and antipyretic drugs are more efficacious in the longer term.

The lack of a clear effect of the addition of tepid sponging to antipyretic medications in the treatment of children with fever suggests that other physical treatments might be of similarly limited use. Removing clothing is less effective than treatments including paracetamol, ${ }^{4}$ and where the two interventions are used together it is likely that most of the benefit will be derived from the effect of the paracetamol. Use of other physical methods, such as fans, in the absence of antipyretic medication might be similarly ineffective.

The influence of the methods of sponging and dosages of paracetamol are harder to assess; however, the lack of any clear, clinically important differences in a diverse set of studies such as these suggests that such influences are minor. Furthermore, such differences as were seen were small enough to be caused by natural temperature fluctuations as a result of circadian rhythms and the errors associated with temperature measurement. The existence of differing methods suggests that there is little agreement as to the best way to apply sponging, although as can be seen, in practice it appears to make little difference within the limits applied in these studies.

\section{Side effects and tolerability}

These are difficult to assess because of the wide variation in the interpretation of what constitutes a side effect. For example, shivering was seen occasionally, ${ }^{235}$ and was rightly considered to be a deleterious effect of the treatment; however, the status of crying is less clear. If crying is seen as a side effect, this might have profound implications for paediatric practice, because many interventions carried out upon infants can result in crying.

One group reported pronounced discomfort in only one patient receiving sponging, but crying was reported in over half of this group 
compared with less than one in 10 in the paracetamol group ${ }^{5}$; others noted that equal numbers of children objected to, and enjoyed, the sponging. ${ }^{4}$ However, there is no evidence of any rebound effect that would result in an increase in temperature when sponging is discontinued.

Although there appears to be no evidence of any medium or long term side effects from either intervention, paracetamol appears to be a better tolerated treatment ${ }^{35}$ and is preferred by parents. ${ }^{34}$ This is important because the care of most infants with fever occurs in the home setting, and parents might be less likely to comply with a treatment that is upsetting for the child, and which they themselves do not like.

\section{Conclusion}

The addition of tepid sponging to paracetamol in the treatment of children offers little advantage over the administration of paracetamol alone in most cases. Although it might result in a slightly faster fall in temperature, this benefit is short lasting. This effect might be of use in some situations, but in general paediatric practice the difference is too small to be of clinical importance, particularly when the time involved in administering the sponging is considered.

Although there is little research looking at other physical methods of antipyresis, these results suggest that they might be equally ineffective because they all rely on similar methods of heat loss. ${ }^{1}$ Although there is doubt as to the need for antipyretic treatments, where they are prescribed, it is suggested that antipyretic drugs be the treatment of choice in most cases. Physical methods should be used only where there are specific indications, or the child finds them of comfort.

1 Sukkar MY, El-Munshid HA, Ardawi MSM. Concise human physiology. Oxford: Blackwell Scientific Publications, 1993.

2 Newman J. Evaluation of sponging to reduce body temperature in febrile infants. Can Med Assoc f 1985;132:641-2.

3 Sharber J. The efficacy of tepid sponge bathing to reduce fever in young children. Am f Emerg Med 1997;15:188-92.

4 Kinmoth AL, Fulton Y, Campbell MJ. Management of feverish children at home. BMf 1992;305:1134-6.

5 Mahar AF, Allen SJ, Milligan P, et al. Tepid sponging to reduce temperature in febrile children in a tropical climate. Clin Pediatr (Phila) 1994;33:227-31.

6 Agbolousu NB, Cuevas LE, Milligan P, Broadhead RL, Brewster D, Graham SM. Efficacy of tepid sponging versus paracetamol in reducing temperature in febrile children. Ann Trop Paediatr 1997;17:283-8. 